\title{
Effects of the Need for Cognitive Closure and guidance on contribution quality in wiki-based learning
}

\author{
Sven Heimbuch ${ }^{1}$ and Daniel Bodemer ${ }^{1}$ \\ ${ }^{1}$ University of Duisburg-Essen, Germany \\ Corresponding author: Sven Heimbuch \\ Email address: sven.heimbuch@uni-due.de
}

\section{ABSTRACT}

Controversies based on opposing points of view and contradictory evidence can be fruitful to trigger individual elaboration processes. However, research showed that many wikis are not necessarily suited to make relevant content salient and thus users need further guidance. In an experimental laboratory study on wikis, we investigated two guidance types in conjunction with the Need for Cognitive Closure: (1) visual markers to highlight controversy status (implicit guidance) and (2) a collaboration script that directs users towards discussions (explicit guidance). We analysed the quality of $N=724$ wiki contributions, namely final edits to an article and corresponding discussion replies. The results show that both guidance types do neither directly affect the user contributions to the wiki nor the learning outcome. In interaction with the individual Need for Cognitive Closure there was a meaningful effect on the quality of discussion contributions, matching previous research findings on the quantitative learning outcomes.

\section{INTRODUCTION AND BACK- GROUND}

Working and learning productively and efficiently in groups is becoming increasingly important (Miller and Hadwin, 2015). Net-based communication can help by maintaining the benefits of offline communication, such as mutual knowledge sharing (McLoughlin, 2002), while reducing disadvantages, such as social inhibitions (Murray, 2003). However, it is not only possible to use net-based environments for communication, but it can also support users in their individual learning processes and (cooperative) knowledge construction (Cress and Kimmerle, 2008). A well-known and popular environment, which offers the possibility of cooperative knowledge construction, are wiki pages and the corresponding discussion pages. These allow participants to participate in existing discussions, i.e. express themselves or even clean up a topic, initiate new discussions about topics that are important to them, and finally make changes to the article, ideally based on the discussions about an article.
Such collaborative article writing is one of the most common group activities (Schlichter et al., 1998). In a wiki environment, it is not unusual or unintended for conflicts or constructive controversies to occur (Johnson et al., 2000b) when the wiki information deviates from one's personal knowledge (Cress and Kimmerle, 2007). This makes it necessary to assimilate or accommodate internally from the user's perspective or externally from the wiki's perspective. This shows that precisely these conflicts can be particularly helpful for the knowledge construction and learning success of the individual (Piaget, 1977). Furthermore, they can also lead to partners being more motivated and using their expertise more effectively (Johnson et al., 2000a).

The Need for Cognitive Closure (NCC) is an important influence on people's behaviour in dealing with controversies. It is the need that people want to find an obvious solution, although it does not always have to be the right solution (Dijksterhuis et al., 1996). People with a high NCC strive to achieve a quick solution and show insecure aver- 
sive behaviour. However, people with a lower NCC tend to prefer finding the best solution to ambiguity (Schumpe et al., 2017; Webster and Kruglanski, 1997). This difference between high and low NCC persons can also be measured in the speed of decisions, as people with high NCCs perceive ambiguity as unpleasant. Consequently, they tend to make decisions more quickly and use less information and anchor heuristics for judgment formation (Schlink and Walther, 2007). In contrast to that, people with a low NCC enjoy the uncertainty of ambiguous situations. Thus, they tend to postpone the decision and prefer weighing and finding more information (Schlink and Walther, 2007). In wiki-based learning settings, individuals with a low NCC are more likely to search purposefully for additional in-depth information about a topic in an ambiguous situation, whereas high NCC persons will more likely process the simplest information at hand. Although there are close ties between the NCC and inter-individual differences in learning and knowledge construction, there are only few studies in technology-enhanced learning addressing this construct.

With increasing complexity of digital learning environments with, it can become helpful that learners are further supported in dealing with controversial and ambiguous information with the help of supplemental Cognitive Group Awareness tools (Bodemer and Buder, 2006). Although minimal tacit guidance for learners has been questioned by others (Kirschner et al., 2006), we do not fully agree to such floccinaucinihilipilification of implicit guidance. Cognitive group awareness tools that are focused on gathering and visualising knowledgerelated contextual cues have been successfully implemented as implicit guidance measures to structure collaborative learning processes (Heimbuch and Bodemer, 2017). Another line of wiki-related research has proposed additional measures of explicit guidance to incorporate in wiki-based learning environments to improve the overall quality of knowledge artefacts and for better coordination processes of students. The implementation of collaboration scripts is one possible explicit guidance measure where the activities of writers and editors within a social system are coordinated and opti- mised. A script is a set of instructions that specifies the group formation, modes of interaction and task management between collaboration partners (Dillenbourg, 2002). Positive effects have been found for scripts with a special focus on article editing and revising that ultimately led to more coherent articles and fewer inaccurate articles (Wichmann and Rummel, 2013). Furthermore, it has also been shown that a certain level of coercion in the implemented collaboration script is recommended to produce content of higher quality (Papadopoulos et al., 2013).

In previous analyses of the underlying study data, we could show that test persons in the experimental study groups (implicit vs. explicit or high vs. low NCC) showed no meaningful differences in the process variables with the log data that we measured (e.g., topic selection, time to contribute, topic reply frequency), as assumed in the hypotheses (Heimbuch and Bodemer, 2018). Likewise, there was no direct effect of the guidance type on the performance in the knowledge test. The in-depth investigation of the interaction of guidance type and NCC confirmed the hypothesised pattern. Evidence suggested that subjects with a low NCC achieved higher test scores with explicit guidance (collaboration script) and accordingly subjects with a relatively high NCC achieved better scores with implicit guidance (controversy awareness highlights). These findings support our claim that implicit guidance provides a quick, non-restrictive way to find a solution, which is preferred by those persons with a high NCC. Explicit guidance can be beneficial to people with a low NCC, as it can support them to find a better solution rather than just the quickest. To complete our previous analyses on the quantitative part, we were left with the question if we can find promising effects on the quality of contributions when we specifically investigate implicit and explicit guidance in the interplay with the Need for Cognitive Closure. Thus, we were interested in two main research questions:

$R Q$ 1: What kind of contributions would we find in either the implicit guidance or the explicit guidance wiki group, and how can these be translated into a quality measure? 
$R Q 2$ : Does the type of guidance directly or indirectly influence the quality of contributions and learning outcomes, and how does the NCC moderate potential effects?

\section{METHOD}

\subsection{Participants and study design}

The $N=181$ participants were mostly undergraduate students at the University of Duisburg-Essen (Germany) in their first semester. Students were recruited via the university's social media channels and on-site. Their age range spanned from 17 to 33 years $(M=20.59, S D=2.59)$. We have randomly assigned students to one of two experimental learning environments about different forms of energy (e.g., nuclear power, fossil fuels, renewable energy). This was either a wiki with added controversy awareness highlights for implicit guidance or a wiki with a collaboration script for explicit guidance. Thus, this variation of wikis was our main independent variable. Furthermore, we have assessed the Need for Cognitive Closure with the German short scale 16-NCCS (Schlink and Walther, 2007) of each participant and used this as a second factor for analytical purposes. Our main dependent variable was the article and discussion quality of each student's contribution. Moreover, we have also measured numerous process variables through data logging and learning outcomes with knowledge tests.

\subsection{Study procedure}

We conducted the experiment in an individual setup with up to four participants at the same time, separated by divider panels. Participants performed all the experiment's stages individually in their own wiki instance. After participants were briefed with standardised written instructions on the computer screen and had given consent to participate in the study, they were first asked a few basic sociodemographics as well as interest in and prior knowledge of the study's subject matter (forms of energy). This was followed by a short mandatory introduction to the specific wiki environment. We asked the participants to click through a mock-up environ- ment with "lorem ipsum" texts to familiarize with the general wiki structure. In addition to the general orientation in a wiki, this tutorial phase also served to familiarize with the specific additions we added to the experimental wikis (controversy highlighting vs collaboration script) to ensure that participants have a common ground about their wiki environment's mechanics (Figure 1).

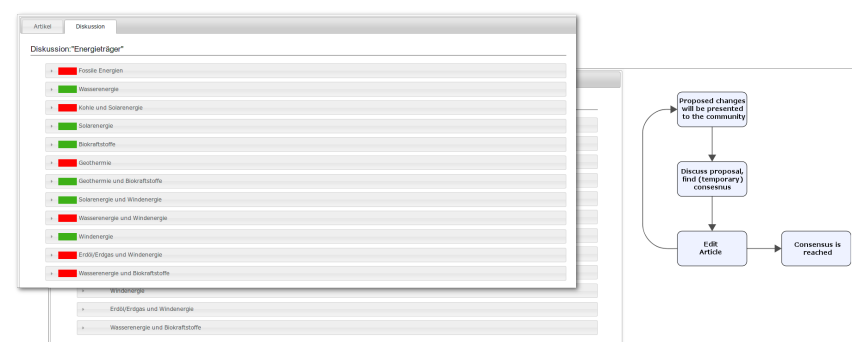

Figure 1. In the floating upper left part of the figure, we illustrate the wiki's talk page table of contents with controversy status highlights for implicit guidance. On the right, we illustrate a collaboration script representation as used for the explicit guidance wiki.

Both groups had the same task of contributing to an initial Wikipedia-like base article about different forms of energy and participating in up to three of the corresponding discussions. Participants received the information that the discussions contain enough arguments and evidence to enrich the original article, since we did not provide any other additional material regarding the subject matter elsewhere. We gave no further instructions on how to start their wiki task (e.g., reading the article or any discussion first) or what kind of reply they should make to a self-selected discussion. This was the experiment's main stage where participants had a loose total time limit of 21 minutes for finishing all article edits and discussion replies. After the time for a contribution phase was up, they the environment automatically prompted them to finish their contributions in the wiki and proceed further. Followed by the wiki contribution stage, we provided them with the questionnaires to determine their individual levels of their Need for Cognitive Closure (16-NCCS). After filling out these questionnaires participants had to answer a multiple-choice test 
about the study's contents. As an additional manipulation check, we asked participants to sum up briefly in open text fields why they have selected certain discussions to comment on and what led to the final decisions for the resulting article edits. Finally, to gain insights about how participants evaluate the additions we made to the wikis we asked them to fill out the User Experience Questionnaire (UEQ) by Laugwitz et al. (2008).

\subsection{Wiki contribution coding}

In total, we had to analyse $N=181$ article edits and $N=543$ replies to the article's discussions. We decided to start our content categorisation deductively with previously discussed categorisations of user types in wikis who participate in the co-evolution of knowledge (Cress and Kimmerle, 2008). The first two categories for edits to the article that we have derived where Accommodators and Assimilators. The former is mainly characterised as a user whose contributions are mostly restructuring and synthesizing tasks, whereas the latter in the simplest form is purely adding new content to the wiki and not caring much about purposeful integration (Majchrzak et al., 2006). With further inductively derived categorisations for article edits, we added four additional categories: Reformulator, Reformulatorshortener, Shortener and No edit. Subsequently, we used these final six article editing categorisations for our quality assignments (Table 1). A second trained coder was asked to categorise and rate a random sample of 12 articles and resulted in a $\kappa=.79$, which is an adequate level of concordance (Mayring, 2015).

As a second step of our quality analyses, we had to work through the discussion replies and assign adequate categories to these contributions. We decided to work fully inductive with the material in multiple iterations, because we had to expect a large diversity in reply content and quality. Finally, we ended up with seven discussion reply categories: No statement, Repetition, Addition, Compromise, Other (related) topic, Personal criticism, Other. Furthermore, we arranged the discussion reply categories in a flat hierarchy. Therefore, we created two higherlevel categories for participants' replies, namely test persons who contributed to the course of the discussion with their comments and those who replied to discussion threads without advancing the discussion by any means (Figure 2).

Table 1: Assignment of categories to article quality ranks (with justifications).

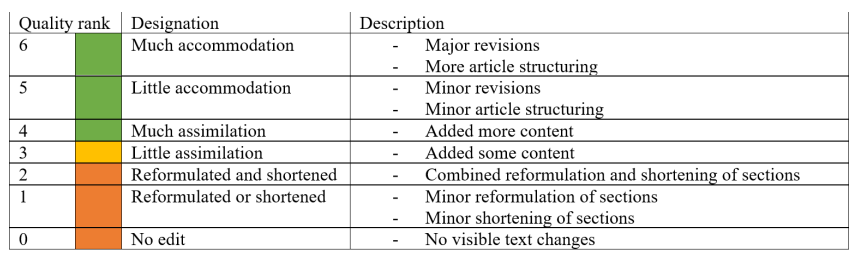

Note. Orange $=$ lower quality, yellow $=$ medium quality, green $=$ higher quality

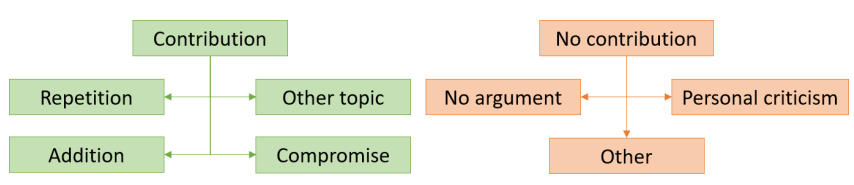

Figure 2. Schematic representation of the hierarchy of the categories into two main categories and a total of seven subcategories.

In contrast to the quality assignments for the final article edits, we assigned quality ranks to discussion patterns. The order in which the categories appeared in the three discussions that each participant had to contribute was irrelevant. In order to obtain a quality score for a participant over the course of three discussion contributions, in a next step, we further examined the patterns occurring in the discussion. As can be seen in Table 2, we assigned the highest quality level (rank 9) to a person who brought three new arguments as Additions into the discussions. A second trained coder was asked to categorise and rate a random sample of 15 discussion contributions and resulted in a $\mathrm{k}=.74$, which is again an acceptable level of concordance.

\subsection{Path analysis}

Since this study had a serial process in the action steps of the test persons (three separate iterations of discussing and editing), a path analysis in form 
Table 2: Assignment of categories to quality ranks for discussion triads.

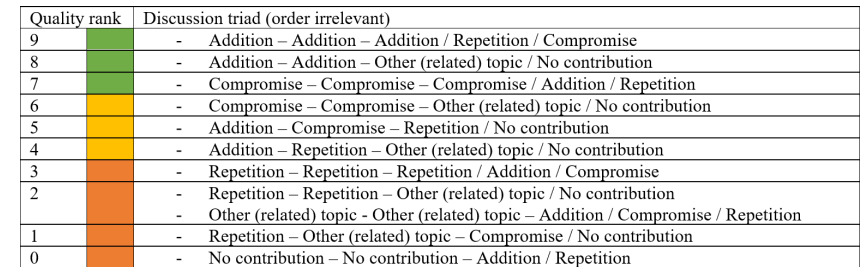

Note. Orange $=$ lower quality, yellow $=$ medium quality, green $=$ higher quality

of a serial mediation offered itself for the investigation of the effects (Hayes, 2018; Hayes et al., 2017). Thus, we investigated the path effects of the quality assignments as serial mediators in a row on the effect of the independent variable (type of additional wiki guidance) on the knowledge test scores as learning outcome, which has been previously analysed in a different context (Heimbuch and Bodemer, 2018).

The score in the knowledge test achieved by the test persons served as the dependent variable. The knowledge test consisted of 18 multiple-choice questions (up to three distractors, at least one attractor) relating to the subject matter of energy sources as presented in the discussions and the wiki article. Furthermore, in order to investigate potential influences of the Need for Cognitive Closure, we performed a moderated serial mediation with discussion quality as first mediator and article edit quality as second mediator. We have chosen this mediator order after investigating the log files and confirming that all participants started with a discussion before an article edit was performed.

\section{RESULTS AND DISCUSSION}

In order to provide a brief insight into the relationship between the quality allocations of the systems, Table 3 presents an excerpt of the results of both category systems side by side. two category systems side by side. Even at first glance, a wide variety of combinations of quality ranks can be determined based on the colour coding of the quality ranks. For example, there are similarities between the two quality assignments, as in the case of test person
97, who contributed on a high-quality level in both cases (both green). A match of the quality assignments can also be found with person 9 (both orange) or 96 (both yellow). But there are also differences, as in the case of person 99, whose contributions to the discussion were classified as high quality (green) but the discussion replies were classified as low quality (orange). Overall, in the whole coded dataset we see a relatively even distribution of ranks and user type classifications across both types of guidance.

Table 3: Sample section of the quality assignments in both category systems side by side.

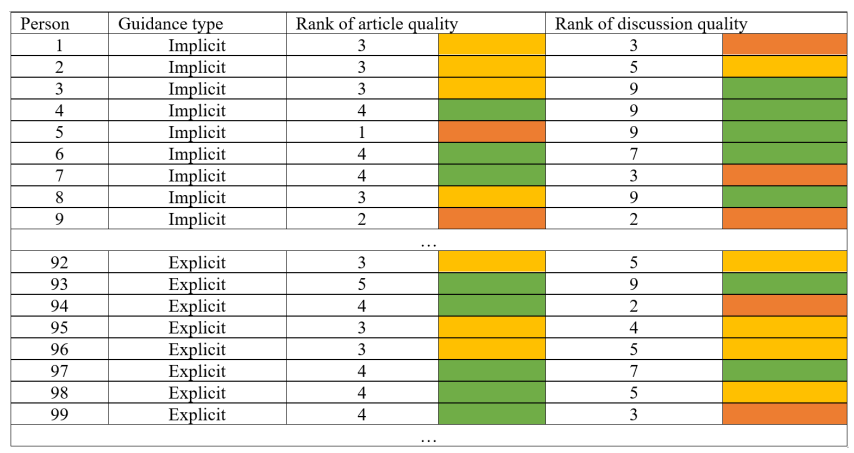

The assigned user categories showed us that the most frequently assigned category was that of assimilators, which translated into a high frequency of medium quality contributions to the final article. We assume that social inhibitions could also exist in such a web-based study setting and that the test person must get used to the situation before he or she is "ready" for collaborative knowledge construction (Cole, 2009; Kump et al., 2013). This may have had an influence on the procedure of the test persons to the extent that they did not want to make any more far-reaching changes to the article. As outlined in Table 3, there are no major quantitative differences between the groups with implicit or explicit support in terms of corresponding quality distribution. This is further supported by the results of the path analysis in the following subsection. The path effect of the guidance type on the article editing quality was very small and far from any acceptable level of significance (see Figure 3, path $d$ ), which means that the guidance type had no meaningful direct 
effect on the final article quality. It should be noted, of course, that since these results were based on a qualitative content analysis, they depend very much on the underlying data. However, since similar categories have already been found in other studies (Majchrzak et al., 2006), this indicates that there is a certain generalisability.

When we further investigated the discussion categorisations, we saw the highest frequencies of discussion replies as additions and repetitions, corresponding to higher and medium quality ranks in our assignments. On the one hand, a high number of persons in the repetition category could be explained by superficial processing that would require a rather small amount of cognitive effort (Vertzberger, 1990) to reiterate the arguments of the other participants and thus to write one's own statement. Therefore, if the subjects had not been sufficiently motivated (Cole, 2009) or involved to engage in high cognitive (Newman et al., 1995), they might have relied on behaviour that required only low cognitive effort. On the other hand, the high number of persons in the category additions could be explained by the fact that at least some of our test persons were interested and motivated to engage in deeper, more complex behaviour and could therefore ultimately participate in higher quality discussions (Newman et al., 1995). In addition, they may have already had previous knowledge or a firm opinion on the subject in question and have written their reply accordingly.

\subsection{Path analysis}

For analysing the potential moderating influences of the NCC on contribution quality and learning outcomes, we have calculated a moderated serial mediation with both discussion quality and article editing quality as mediating variables. These serial mediations were calculated using Model 85 in PROCESS (v3.1) for SPSS (Hayes, 2018). Figure3 shows the results of the paths in this model where we show that only one of the unmoderated paths has become significant, namely the path from the article editing quality to the knowledge test score with a regression weight of $c=.51, p=.007$. From this it can be concluded that there is a meaningful regression of the article editing quality on the knowledge test score, translating into a test score improvement of approximately 0.5 for each raise in the quality rank. Furthermore, one of the paths moderated by the NCC in this analysis was identified to be of further interest. We found a moderating effect of the $\mathrm{NCC}$ on the connection between the guidance type and discussion quality, $g=-.09, p=.023$.

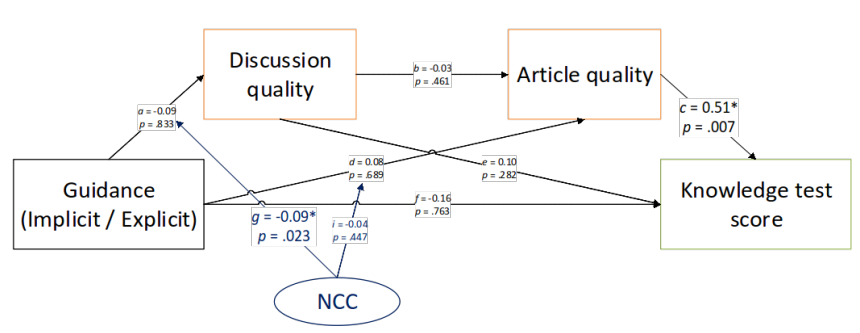

Figure 3. Representation of the path analysis as moderated serial mediation.

In addition to the above model, we further investigated the simple slopes of the moderations on the paths $a$ and $d$ (Figure4). Implicit guidance together with a high NCC and explicit guidance in conjunction with a low NCC can lead to a higher quality of discussion contributions. This matches to the results that were previously presented in the quantitative analyses of the process variables and the learning outcomes (Heimbuch and Bodemer, 2018). This finding can be explained by theories on the NCC and backed by empirical evidence, where persons with a higher NCC want to find rather quick solutions and tend to prefer simpler signals and cue heuristics, whereas low NCC persons enjoy to find better answers and solutions through information seeking and more elaborate discussions (Schlink and Walther, 2007; Webster and Kruglanski, 1994, 1997). The same pattern can also be seen for the article editing quality, although to a much weaker extent. It is reasonable to assume that the effect on this article editing is less obvious, because the NCC refers to dealing with controversies and decisionmaking situations (Dijksterhuis et al., 1996), which were not necessarily present during the article editing phase. That could be a valid reason why the NCC level had no meaningful moderating effect on the article editing slope. 

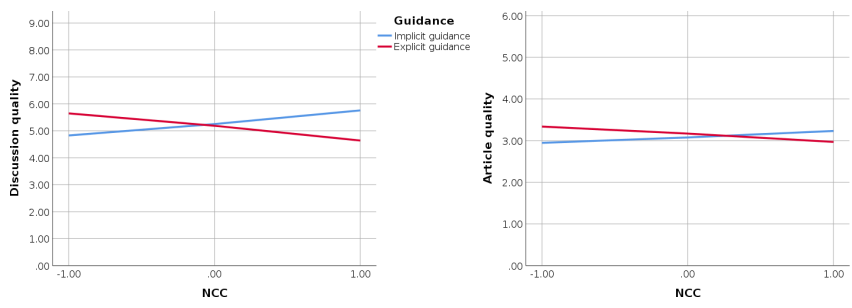

Figure 4. Representations of the moderator effects of the NCC on both quality rank assignments. $-1=$ low NCC; $1=$ high NCC

\section{CONCLUSIONS}

Qualitative content analysis itself was often criticized because of the restriction to rather fixed categories and that this would take the focus from the wholeness of the texts and direct it to paraphrases in the text (Ramsenthaler, 2013). This criticism, however, is debatable and has been refuted by others, since the categories would be re-examined and re-evaluated repeatedly (Mayring, 2014). In our analysis, we categorised entire articles and discussion contributions, which in turn ensured a more holistic view of the text. Thus, we think that through our analytic method, we were able to validly categorise and assign quality ranks to the wiki contributions. Our main finding, that stands in line with previously reported findings on quantitative data (Heimbuch and Bodemer, 2018), is that a generally useful type of additional guidance for technologyenhanced learning may not be enough. Depending on the task, it can be necessary to consider adequate individual personality variables. In this case the Need for Cognitive Closure was valid research subject, since we were explicitly interested in the processing of ambiguous information and if people produce better learning and knowledge construction outcomes when guided either implicitly or explicitly. In future analyses, it might be interesting to consider the constellations of both categorisation systems, as outlined in Table 3, and the type of provided guidance in order to take a closer look at possible correlations between these two variables. In addition, the test persons in this study had the opportunity to leave comments about their contributions to the discussion and choice of topic. For future analyses, it may be interesting to determine how the selection of topics was justified and whether there was a correlation between the justification and the contribution to the discussion.

\section{REFERENCES}

Bodemer, D. and Buder, J. (2006). Supporting collaborative learning with augmented group awareness tools. In Sun, R. and Miyake, N., editors, Proceedings of the Twenty-Eighth Annual Conference of the Cognitive Science Society, pages 77-82, Mahwah, NJ. Lawrence Erlbaum.

Cole, M. (2009). Using Wiki technology to support student engagement: Lessons from the trenches. Computers \& Education, 52(1):141-146.

Cress, U. and Kimmerle, J. (2007). A theoretical framework of collaborative knowledge building with wikis: A systemic and cognitive perspective. In Proceedings of the 8th Iternational Conference on Computer Supported Collaborative Learning, pages 156-164, New Brunswick, NJ. International Society of the Learning Sciences.

Cress, U. and Kimmerle, J. (2008). A systemic and cognitive view on collaborative knowledge building with wikis. International Journal of Computer-Supported Collaborative Learning, 3(2):105.

Dijksterhuis, A., van Knippenberg, A., Kruglanski, A. W., and Schaper, C. (1996). Motivated Social Cognition: Need for Closure Effects on Memory and Judgment. Journal of Experimental Social Psychology, 32(3):254-270.

Dillenbourg, P. (2002). Over-scripting CSCL: The risks of blending collaborative learning with instructional design. In Kirschner, P. A., editor, Three worlds of CSCL. Can we support CSCL?, pages 61-91. Heerlen, Open Universiteit Nederland.

Hayes, A. F. (2018). Introduction to mediation, moderation, and conditional process analysis: a regression-based approach. Methodology in the social sciences. Guilford Press, New York, second edition edition.

Hayes, A. F., Montoya, A. K., and Rockwood, N. J. (2017). The analysis of mechanisms and their contingencies: PROCESS versus structural equa- 
tion modeling. Australasian Marketing Journal (AMJ), 25(1):76-81.

Heimbuch, S. and Bodemer, D. (2017). Controversy awareness on evidence-led discussions as guidance for students in wiki-based learning. The Internet and Higher Education, 33(2017):1-14.

Heimbuch, S. and Bodemer, D. (2018). Interaction of guidance types and the Need for Cognitive Closure in wiki-based learning. PeerJ, 6:e5541.

Johnson, D. W., Johnson, R. T., and Smith, K. A. (2000a). Constructive controversy: The educative power of intellectual conflict. Change: The Magazine of Higher Learning, 32(1):28-37.

Johnson, D. W., Johnson, R. T., and Tjosvold, D. (2000b). Constructive controversy: The value of intellectual opposition. In Deutsch, M., Coleman, P. T., and Marcus, E. C., editors, The handbook of conflict resolution: Theory and practice, pages 65-85. Jossey-Bass, San Francisco, CA.

Kirschner, P. A., Sweller, J., and Clark, R. E. (2006). Why Minimal Guidance During Instruction Does Not Work: An Analysis of the Failure of Constructivist, Discovery, Problem-Based, Experiential, and Inquiry-Based Teaching. Educational Psychologist, 41(2):75-86.

Kump, B., Moskaliuk, J., Dennerlein, S., and Ley, T. (2013). Tracing knowledge co-evolution in a realistic course setting: A wiki-based field experiment. Computers \& Education, 69:60-70.

Laugwitz, B., Held, T., and Schrepp, M. (2008). Construction and Evaluation of a User Experience Questionnaire. In Holzinger, A., editor, HCI and Usability for Education and Work, number 5298 in Lecture Notes in Computer Science, pages 63-76. Springer Berlin Heidelberg.

Majchrzak, A., Wagner, C., and Yates, D. (2006). Corporate wiki users: results of a survey. In Proceedings of the 2006 international symposium on Wikis - WikiSym '06, page 99, Odense, Denmark. ACM Press.

Mayring, P. (2014). Qualitative content analysis: theoretical foundation, basic procedures and software solution. Klagenfurt.

Mayring, P. (2015). Qualitative Content Analysis: Theoretical Background and Procedures. In Bikner-Ahsbahs, A., Knipping, C., and Presmeg,
N., editors, Approaches to Qualitative Research in Mathematics Education: Examples of Methodology and Methods, Advances in Mathematics Education, pages 365-380. Springer Netherlands, Dordrecht.

McLoughlin, C. (2002). Computer supported teamwork: An integrative approach to evaluating cooperative learning in an online environment. Australasian Journal of Educational Technology, 18(2).

Miller, M. and Hadwin, A. (2015). Scripting and awareness tools for regulating collaborative learning: Changing the landscape of support in CSCL. Computers in Human Behavior.

Murray, M. H. (2003). Managing teamwork online. In A forum for engaging, designing, assessing, and collaborating in online learning and teaching, pages 75-82, Brisbane, Australia. Queensland University of Technology.

Newman, D. R., Webb, B., and Cochrane, C. (1995). A Content Analysis Method to Measure Critical Thinking in Face-to-Face and Computer Supported Group Learning. Interpersonal Computing and Technology, 3(2):56-77.

Papadopoulos, P., Demetriadis, S., and Weinberger, A. (2013). 'Make it explicit!': Improving collaboration through increase of script coercion: 'Make it explicit!'. Journal of Computer Assisted Learning, 29(4):383-398.

Piaget, J. (1977). The development of thought: equilibration of cognitive structures. Viking Press. Google-Books-ID: PsM_AQAAIAAJ.

Ramsenthaler, C. (2013). Was ist „Qualitative Inhaltsanalyse?“‘. In Schnell, M., Schulz, C., Kolbe, H., and Dunger, C., editors, Der Patient am Lebensende, pages 23-42. Springer Fachmedien Wiesbaden, Wiesbaden.

Schlichter, J., Koch, M., and Bürger, M. (1998). Workspace awareness for distributed teams. In Goos, G., Hartmanis, J., van Leeuwen, J., Conen, W., and Neumann, G., editors, Coordination Technology for Collaborative Applications, volume 1364, pages 199-218. Springer Berlin Heidelberg, Berlin, Heidelberg.

Schlink, S. and Walther, E. (2007). Kurz und gut: Eine deutsche Kurzskala zur Erfassung des 
Bedürfnisses nach kognitiver Geschlossenheit. Zeitschrift für Sozialpsychologie, 38(3):153-161. Schumpe, B. M., Brizi, A., Giacomantonio, M., Panno, A., Kopetz, C., Kosta, M., and Mannetti, L. (2017). Need for Cognitive Closure decreases risk taking and motivates discounting of delayed rewards. Personality and Individual Differences, 107:66-71.

Vertzberger, Y. Y. (1990). The world in their minds: Information processing, cognition, and perception in foreign policy decisionmaking.

Webster, D. M. and Kruglanski, A. W. (1994). Individual differences in need for cognitive closure. Journal of Personality and Social Psychology, 67(6):1049-1062.

Webster, D. M. and Kruglanski, A. W. (1997). Cognitive and Social Consequences of the Need for Cognitive Closure. European Review of Social Psychology, 8(1):133-173.

Wichmann, A. and Rummel, N. (2013). Improving revision in wiki-based writing: Coordination pays off. Computers \& Education, 62:262-270.

\section{ACKNOWLEDGEMENTS}

We would like to thank Manon-Lea Laudien, B.Sc. for preparing the data in the course of her Bachelors' thesis. 